\title{
Clinical and endoscopic profile of patients with Mallory- Weiss tears
}

\author{
Subash Bhattarai', Khus Raj Dewan², Gaurav Shrestha ${ }^{3}$, Bhanumati Saikia Patowary ${ }^{4}$ \\ 1,2,3 ${ }^{3}$ ecturer, ${ }^{4}$ Professor, Department of Medical Gastroenterology, College of Medical Sciences and Teaching Hospital, \\ Chitwan, Nepal
}

Background: Mallory-Weiss tear is mainly located across the gastro-esophageal mucosal junction and presents with upper gastro-intestinal bleed of less severity. Haemorrhage frequently ceases spontaneously. Aims and Objective: This study was undertaken to establish the incidence, clinical and endoscopic profile of patients with Mallory- Weiss tear. Materials and Methods: Six hundred patients presenting with acute upper gastrointestinal (UGI) bleed were included in the study. All patients underwent UGI endoscopy after achieving haemodynamic stability, usually within 24 hours. Cases with Mallory Weiss tear were identified and their clinical presentation, endoscopic findings, outcomes during hospitalization including rebleeding and mortality were studied. Results: Twenty eight $(4.6 \%)$ patients out of six hundred acute upper gastro-intestinal bleed were diagnosed with Mallory-Weiss tear. Fourteen (50\%) patients gave history of prior vomiting or preceeding retching. Only two patients $(7.1 \%)$ presented with shock. The most common co-morbid condition was excessive alcohol consumption. Eighteen $(64.3 \%)$ patients had no active bleeding during UGI endoscopy. All patients except four presented with a single tear with mean length of two $\mathrm{cms}$. Blood transfusion was needed in eight (28.6\%) patients only. Only one patient had re-bleeding. Average hospital admission was three days. No mortality was recorded in 7 days and 28 days of follow ups. Conclusions: Mallory-Weiss tear is not an uncommon cause of UGI bleeding nowadays and its incidence is rising every year. Hemorrhage frequently ceases spontaneously and conservative management is sufficient in many instances. When endoscopic findings reveal active haemorrhage, various endoscopic hemostatic techniques can be used.

Key words: Upper gastro-intestinal bleed, Mallory-Weiss tear, Endoscopy

\section{INTRODUCTION}

Mallory and Weiss first described gastro-oesophageal tears causing gastrointestinal bleeding in 15 alcoholic patients in 1929. ${ }^{1}$ Mallory-Weiss tear (MWT) is a laceration (tear) in the esophageal mucous membrane (inner lining) at the site where the distal esophagus meets the proximal stomach. The lacerations often lead to bleeding from submucosal arteries. The prevalence of Mallory-Weiss tear is reported to be approximately $5 \%$ of patients suffering acute upper gastrointestinal bleeding. ${ }^{2}$

\section{Access this article online}

Website:

http://nepjol.info/index.php/AJMS

DOI: 10.3126/ajms.v8i6.18102

E-ISSN: 2091-0576

P-ISSN: 2467-9100 
tear presenting with upper gastrointestinal (UGI) bleed. This study may prove helpful in establishing clinical and endoscopic correlations and guiding in management of these patients.

\section{MATERIALS AND METHODS}

This observational, cross-sectional, prospective hospital based study was carried out in the department of medical gastroenterology at College of Medical Sciences and Teaching Hospital, Nepal from January 2015 to June 2017.

Six hundred patients with acute upper GI bleeding presenting with either haematemesis and/or melaena were enrolled for the study. Data considering demographic variables, clinical features, bleeding characteristics were collected and alongside blood investigations like complete blood count, platelets count, blood grouping, liver function test, prothrombin time/international normalized ratio (PT/ INR), coagulation profile and others were collected.

Each patient underwent endoscopic investigation by standard flexible gastroduodenal endoscope (PENTAX EPK 700, PENTAX JAPAN Inc) after achieving haemodynamic stability, usually within 24 hours. In cases, when multiple lesions were found in UGI endoscopy, the lesion with active bleeding or recent stigmata of haemorrhage was considered the cause of bleeding.

Pharmacologic treatment with intravenous proton pump inhibitors and endoscopic treatment with injection epinephrine were used to maintain haemostatsis. Cases with Mallory Weiss tear were identified and their clinical presentation, endoscopic findings, outcomes during hospitalization including rebleeding and mortality were monitored.

Inclusion criteria: All patients with acute upper GI bleed

\section{Exclusion criteria}

1. Patients on warfarin or other anticoagulants

2. Critically ill patients and those who fail to give consent

The study was approved and verified by Institutional Review Committee and the work was in accordance with the rules and regulations laid down by the Institutional Review Committee.

\section{Statistics}

Data were collected on a structured proforma covering the relevant subjects of the study and data entry followed by analysis was done in Statistical Packages for the Social Sciences (SPSS) version 20. All categorical data were expressed in percent and absolute number. All numerical continuous data were expressed in mean \pm standard deviation.

\section{RESULTS}

Six hundred patients aged between 14 and 88 years of age presentation with UGI bleeding; 410 male $(68.3 \%)$ and 190 female (31.7\%) were enrolled in the study. The most common cause of UGI bleed was gastro-oesophageal varices $(40.7 \%)$ followed by peptic ulcer disease $(31.7 \%)$. Non variceal causes of UGI bleed were duodenal ulcer $(18.6 \%)$, gastric ulcer $(13.1 \%)$ followed by portal hypertension gastropathy $(6.7 \%)$,erosive mucosal lesions $(12.7 \%)$, Mallory- Weiss tear (4.6\%) and GI malignancy (3.6\%) respectively as shown in Table 1.

Twenty eight cases $(4.6 \%)$ of patients with upper gastrointestinal bleed were identified with Mallory Weiss tear. Patients with Mallory Weiss tear were aged between 19 and 75 years of age (mean $45.8 \pm 16.16$ years). The mean ages of male and female patients were 49.6 and 39.8 years respectively $(\mathrm{M}: \mathrm{F}=1.6: 1)$.

Fourteen $(50 \%)$ patients gave history of prior vomiting or preceding retching. These symptoms were commonly seen in alcoholic cirrhosis, alcohol dependent patients and in pregnant women most commonly. Eighteen $(64.3 \%)$ patients presented with only haematemesis, three $(10.7 \%)$ with malena; and seven $(25 \% \%)$ with haematemesis and malena.Only two patients (7.1\%) presented with shock. Mean haemoglobin at presentation was $11.2 \pm 1.63 \mathrm{gm} \%$ (range of $8 \mathrm{gm} \%$ to $14 \mathrm{gm} \%$ ). Only five patients presented with haemoglobin $\leq 10 \mathrm{gm} \%$. Mean platelets count was 3, 37,000 $\pm 1,24,540 / \mathrm{mm} 3$ with a minimum of $90,000 / \mathrm{mm}^{3}$ and a maximum of $5,10,000 / \mathrm{mm}^{3}$.

\begin{tabular}{lc}
$\begin{array}{l}\text { Table 1: Distribution of subjects according to } \\
\text { aetiology of UGI bleed }\end{array}$ \\
\hline Aetiology of UGI bleed & $\mathbf{N = 6 0 0 ( \% )}$ \\
\hline Gastro-oesophageal varices & $244(40.7)$ \\
Oesophageal Varices & $240(40)$ \\
Gastric varices & $4(0.7)$ \\
Peptic ulcer diseases & $190(31.7)$ \\
Duodenal ulcer & $112(18.6)$ \\
Gastric ulcer & $78(13.1)$ \\
Portal hypertensive & $40(6.7)$ \\
gastropathy & \\
Erosive mucosal lesions & $76(12.7)$ \\
Erosive gastritis & $39(6.6)$ \\
Erosive oesophagitis & $23(3.8)$ \\
Erosive duodenitis & $14(2.3)$ \\
Mallory Weiss tear & $28(4.6)$ \\
GI malignancies & $22(3.6)$ \\
Gastric carcinoma & $14(2.3)$ \\
Oesophageal Carcinoma & $8(1.3)$ \\
\hline
\end{tabular}


In the present study, the common co-morbid conditions were alcoholic cirrhosis of liver in four patients, alcohol dependence in three, pregnancy with vomiting in four, chronic kidney disease in three. Three patients had systemic hypertension, two with diabetes mellitus, one case of ischemic heart disease and five patients had no co morbid conditions. Two cases had mucosal tear during UGI endoscopy done for evaluation of gastro-oesophageal reflux disease in the present study.

Mean complete rockall score after UGI endoscopy was two with a range from zero to five. Twenty four patients $(37.5 \%)$ had Rockall score $\leq 3$. Four patients had Rockall score of four and only two patients had maximum Rockall score of five. Four patients had oozing of blood from the tear; six patients had an adherent clot. Eighteen (64.3\%) patients had no active bleeding during UGI endoscopy. All patients except four presented with a single tear with mean length of two cms. Four patients presented with two mucosal tears. Two patients were of alcohol dependence, one was pregnant lady and another was chronic kidney disease (CKD) patient with uraemia to present with two mucosal tears.

Coexisting mucosal lesions, but not source of bleeding was also identified in thirteen cases. Four patients had portal hypertensive gastropathy, four cases had gastric ulcer, one case had duodenal ulcer, three patients had erosive esophagitis and a single case had erosive gastroduodenitis.

All patients were treated with ulcer healing agents and intravenous proton pump inhibitor. One patient with oozing of blood was also treated with injection epinephrine that brought hemostasis.

Blood transfusion was needed in eight $(28.6 \%)$ patients only. Four patients were transfused two pints of blood and the rest four were transfused a single pint of blood.

Only one patient had re-bleeding. The only cases that rebleed occurred in a pregnant lady on $5^{\text {th }}$ day who was discharged on second day of hospital admission. Average hospital admission was three days with a minimum of one day and maximum of seven days. Patients with cirrhosis of liver, alcohol dependence and diabetes ketoacidosis cases were admitted for $\geq 5$ days for management of their coexisting illness. No mortality was recorded in 7 days and 28 days of follow ups.

\section{DISCUSSION}

Twenty eight $(4.6 \%)$ patients with upper gastrointestinal bleed were identified with Mallory Weiss tear (MWT).
Dwivedi et al, ${ }^{4}$ in India, reported $15.5 \%$ of UGI bleed to have MWT. Knauer et a $\mathrm{l}^{5}$ studied 528 upper gastrointestinal bleeders and incidence of MWT was $11 \%$. Endoscopic diagnoses of MWT were made in $12 \%$ and $10.3 \%$ by Akhtar et $\mathrm{al}^{6}$ and Sugawa et $\mathrm{al}^{7}$ respectively. Watts et $\mathrm{al}^{8}$ reported an incidence of $4.5 \%$, similar to the present study. Yin et al ${ }^{9}$ reported even lower incidence of 3.08\%.

Patients were aged between 19 years and 75 years of age (mean 45.8 years) in the present study. The mean ages were 49.6 years in male and 39.8 years in female respectively. Knauer et a ${ }^{5}$ studied patients with mean ages of 45.2 years and 49.4 years among male and female respectively. Male predominance of $60.1 \%$ was observed in the present study whereas, Knauer et al5 documented even higher male predominance of $77.4 \%$.

The common precipitating factors of the MWT include; vomiting, coughing, hiccups, straining during defecation and lifting heavy objects, blunt abdominal injury, and epileptic convulsions. Esophagogastroduodenoscopy (EGD) as well as bowel preparation with polyethylene glycol lavage for colonoscopy has also been described. ${ }^{10}$

Conditions that predispose to Mallory-Weiss tears have beed established; like presence of hiatal hernia, excessive alcohol use, pregnancy with hyperemesis gravidarum, vomiting due to peptic ulcer and other mucosal diseases. ${ }^{10,11}$ The common co-morbid conditions in the present study were alcoholic cirrhosis of liver in four patients, alcohol dependence in three, pregnancy with vomiting in four, CKD in three. Three patients had systemic hypertension, two with diabetes mellitus, one case of ischemic heart disease and five patients had no co morbid conditions. Two cases had mucosal tear during UGI endoscopy done for evaluation of gastro-oesophageal reflux disease in the present study.

Fourteen patients (50\%) in the present study gave history of prior vomiting or preceding retching. These symptoms were commonly seen in alcoholic cirrhosis, alcohol dependent patients and in pregnant women most commonly. According to Dwivedi et al ${ }^{4} 41 \%$ patients with MWT had no antecedent nausea, retching, abdominal pain or vomiting. Symptoms of emesis or retching preceded actual upper gastrointestinal bleeding in 43 of 58 (74\%) patients according to study by Knauer et al. ${ }^{5}$ Excessive alcohol intake alone was noted in 23 of $58(40 \%)$ cases in that study. However, more than half of the patients with MWT did not have a preceding history of retching or vomiting according to Knauer et al. ${ }^{5}$ There was no antecedent explanation for the tear, such as nausea, retching, abdominal pain, or vomiting in 12 out of 28 patients $(42.9 \%)$ according to Harris et al. ${ }^{12}$ 
Only two patients $(7.1 \%)$ in the present study had shock at admission. Akhtar et $\mathrm{al}^{6}$ reported $31 \%$ of patients with MWT with shock.

All patients except four presented with a single tear with mean length of $2 \mathrm{cms}$ and a maximum of $3 \mathrm{cms}$. Study by Knauer et $\mathrm{al}^{5}$ described mucosal lacerations up to $4 \mathrm{~cm}$ in length with an average length of $1.5 \mathrm{~cm}$. Four (14.3\%) patients; two with alcohol dependent, a single pregnant lady and another chronic kidney disease with uremia presented with double lacerations. Single lacerations were present in 47 , double lacerations in 6 , triple in 4 , and one patient had four lacerations in the study of 58 patients by Knauer et al. ${ }^{5}$ Weaver et al. ${ }^{13}$ observed a a higher $27 \%$ of cases presenting with two or more mucosal tears.

Hiatal hernia has been described in literatures to be associated with MWT. Hiatal hernia was present in 15 cases $(53.5 \%)$ in the present study. Knauer et $\mathrm{al}^{5}$ described 72 $\%$ of the cases with hiatal hernia. Sato et $\mathrm{al}^{14}$ observed increased incidence of hiatus hernia in Mallory-Weiss tear than that in the control group. However, in a recent publication by Corralet a ${ }^{15}$ hiatal hernia was more common in controls, and no significant difference was seen in a multivariate analysis. This recent publication is in contrary to previous publications. ${ }^{5,14}$

Coexisting mucosal lesions, but not source of bleeding was also identified in 13 cases (46.4\%) in the present study. Four patients had portal hypertensive gastropathy, four cases had gastric ulcer, one case had duodenal ulcer, three patients had erosive esophagitis and a single case had erosive gastroduodenitis. Knauer et $\mathrm{al}^{5}$ reported a higher forty-eight of $58(83 \%)$ cases with additional mucosal abnormalities on endoscopy most commomly exudative esophagitis followed by esophageal varices, gastric and/or duodenal erosions, acute gastritis, and ulcers.

Approximately $24 \mathrm{hr}$ after an acute bleed secondary to a Mallory-Weiss laceration, the lesion can appear as a superficial linear ulcer with complete healing occurring frequently in the subsequent 48 to 72 hours. ${ }^{4}$ Patients bleeding with MWT may have normal UGI endoscopy if performed after 72 hours.

All patients were treated with ulcer healing agents and intravenous proton pump inhibitor. One patient with oozing of blood was also treated with injection epinephrine that brought hemostasis.

Conservative medical treatment, local injection, hemoclipping, or multipolar electrocoagulation produced primary hemostasis in $87.5 \%(14 / 16)$ of patients in the study conducted by Yin et al. ${ }^{9}$
Blood transfusion was needed in eight patients $(28.5 \%)$ only. Four patients were transfused two pints of blood and the rest four were transfused a single pint of blood. Watts et al ${ }^{8}$ observed a higher $61 \%$ of patients with MWT required blood transfusion. Mean transfusion requirements were 2.6 units of packed cells in the study by Harris et a ${ }^{12}$ and $26.5 \%$ received four or more units In the study by Knauer et $\mathrm{al}^{5}$ blood transfusion was required in even higher $75 \%$ (40 out of 58) patients; 14 patients received one to two units; 24 received three to seven units; and 6 required more than seven units of blood.

When conservative measures for hemostasis fail, patients can be managed with endoscopic epinephrine injection, hemoclipping and band ligation. Park et a ${ }^{16}$ in their study found no difference in the efficacy or the safety of band ligation vs. epinephrine injection for the treatment of actively bleeding MWT. Huang et al. ${ }^{17}$ have described that endoscopic hemoclipping placement and endoscopic epinephrine injection are equally effective and safe for management of actively bleeding in Mallory -Weiss tear, even in patients with shock or comorbid diseases. Yamaguchi et al. ${ }^{18}$ reported efficacy and safety of hemoclipping performed in 26 out of 58 patients with MWT. Complete haemostatis was achieved and no any complications, recurrent bleeding, or deaths were observed.

No surgical intervention was needed in the current study. Of the 58 patients, five $(8.6 \%)$ required surgical intervention in the study by Knauer et al..$^{5}$

Only one $(5.6 \%)$ patient presented with rebleeding. The only case of rebleed was seen in a pregnant lady on $5^{\text {th }}$ day who was discharged on second day of hospital admission. Rebleeding within 30 days was seen in a higher $12 \%$ of patients in the study by Akhtar et al. ${ }^{6}$

Average hospital admission was three days with a minimum of 1 day to a maximum of 7 days. Patients with cirrhosis of liver, alcohol dependence and diabetes ketoacidosis cases were admitted for $\geq 5$ days for management of their coexisting illness. Most of the patients with MWT (80\%) according to Akhtar et $\mathrm{al}^{6}$ had an uneventful and short hospital stay (range of 1 to 4 days).

No mortality was recorded in 7 days and 28 days of follow ups in the present study. Although the majority of patients with MWT have a benign course, fatal outcomes have also been described in literatures. The mortality rate was $9.7 \%$ according to Fujisawa et al. ${ }^{19}$ The patients with fatal outcome were identified with advanced age,shock on arrival, lower hemoglobin level, more prolonged prothrombin time, higher AST and ALT levels, detection of 
exposed vessels on endoscopy and with higher frequency of rebleeding. Akhtar et al. ${ }^{6}$ documented an overall mortality of 10\%. Endoscopic hemostasis failure and associated comorbidities were identified as the prognostic indicators. Thirty day mortality was observed in 4 out of $34(11.7 \%)$ patients with MWT in their study by Harris et al ${ }^{12}$ Patients who died had other endoscopic abnormalities, such as ulcers or varices, and all had an alcohol history according to Harris et al. ${ }^{12}$

\section{CONCLUSIONS}

Mallory-Weiss tear is not an uncommon cause of UGI bleeding nowadays and occurs due to mucosal tear in gastro-oesophageal junction. Hemorrhage frequently ceases spontaneously and conservative management is sufficient in many instances. The present study showed most of the patients could be managed conservatively. MWT presents with UGI bleed of less severity usually and patients may be discharged in a day or two. The findings of the study is limited with a relatively small sample size of patients with Mallory-Weiss tears. Literatures have suggested that sometimes even patients with MWT can present with profuse bleeding, in shock and patients may require intensive care and interventions. When endoscopic findings reveal active haemorrhage, various endoscopic hemostatic techniques can be used like epinephrine injection, hemoclip application, and band ligation.UGI endoscopies as earlier as possible can detect more cases of Mallory-Weiss tears.

\section{REFERENCES}

1. Mallory GK and Weiss $S$. Hemorrhages from lacerations of the cardiac orifice of the stomach due to vomiting. Am J Med Sci 1929; 178:506-515.

2. Michel L, Serrano A and Malt RA. Mallory-Weiss syndrome: evolution of diagnostic and therapeutic patterns over two decades. Ann Surg 1980; 192(6):716-721.

3. Tanabe $S$ and Saigenji K. Mallory-Weiss syndrome. Nihon Rinsho 1998; 56(9):2332-2335.
4. Dwivedi M and Misra SP. Mallory-Weiss syndrome: clinical features and management. J Assoc Physicians India 1999; 47(4):397-399.

5. Knauer CM. Mallory-Weiss syndrome. Characterization of 75 Mallory-Weiss lacerations in 528 patients with upper Gastrointestinal hemorrhage. Gastroenterology 1976; 71(1):5-8.

6. Akhtar AJ and Padda MS. Natural history of Mallory-Weiss tear in African American and Hispanic patients. J Natl Med Assoc 2011; 103(5):412-415.

7. Sugawa C, Benishek D and Walt AJ. Mallory-Weiss syndrome. A study of 224 patients. Am J Surg 1983; 145(1):30-33.

8. Watts HD and Admirand WH. Mallory-Weiss Syndrome. A Reappraisal JAMA 1974; 230(12):1674-1675.

9. Yin A, Li Y, Jiang Y, Liu J and Luo H. Mallory-Weiss syndrome: clinical and endoscopic characteristics. Eur J Intern Med 2012; 23(4):92-96.

10. Montalvo RD and Lee M. Retrospective analysis of iatrogenic Mallory-Weiss tears occurring during upper gastrointestinal endoscopy. Hepatogastroenterology 1996; 43: 174-177.

11. Dagradi AE, Broderick JT, Juler G, Wolinsky S and Stempien SJ. The Mallory-Weiss syndrome and lesion. A study of 30 cases. Am J Dig Dis 1966; 11(9): 710-721.

12. Harris JM and DiPalma JA. Clinical significance of MalloryWeiss tears. Am J Gastroenterol 1993; 88(12):2056-2058.

13. Weaver DH, Maxwell JG and Castleton KB. Mallory-Weiss syndrome. American Journal of Surgery 1969; 118(6): 887-892.

14. Sato H, Takase $S$ and Takada $A$. The association of esophageal hiatus hernia with Mallory-Weiss syndrome. Gastroenterol Jpn 1989; 24(3):233-238.

15. Corral JE, Keihanian T, Kröner PT, Dauer R, Lukens FJ and Sussman DA. Mallory Weiss syndrome is not associated with hiatal hernia: a matched case-control study. Scand J Gastroenterol 2017; 52(4):462-464.

16. Park CH, Min SW, Sohn YH, Lee WS, Joo YE, Kim HS, et al. A Prospective, Randomized Trial of Endoscopic Band Ligation vs. Epinephrine Injection for Actively Bleeding Mallory-Weiss Syndrome. Gastrointest Endosc 2004; 60: 22-27.

17. Huang SP, Wang HP, Lee YC, Lin CC, Yang CS, Wu MS, et al. Endoscopic Hemoclip Placement and Epinephrine Injection for Mallory-Weiss Syndrome with Active Bleeding. Gastrointest Endosc 2002; 55(7):842-846.

18. Yamaguchi $\mathrm{Y}$, Yamato $\mathrm{T}$, Katsumi $\mathrm{N}$, Morozumi $\mathrm{K}$, Abe $\mathrm{T}$, Ishida $\mathrm{H}$, et al. Endoscopic hemoclipping for upper $\mathrm{Gl}$ bleeding due to Mallory-Weiss syndrome. Gastrointest Endosc 2001; 53(4):427-430.

19. Fujisawa N, Inamori M, Sekino Y, Akimoto K, lida H, Takahata A, et al. Risk factors for mortality in patients with Mallory-Weiss syndrome. Hepatogastroenterology 2011; 58(106):417-420.

\footnotetext{
Authors Contribution:

SB- Concept and design of the study, reviewed the literature, manuscript preparation and critical revision of the manuscript; KRD- Concept, collected data and review of literature, statistically analyzed and interpreted, prepared first draft of manuscript and critical revision of the manuscript; GS- Conceptualized study, literature search, data collection; BSP- Concept of study, review of study, editing.

Orcid ID:

Dr. Subash Bhattarai: (1) http://orcid.org/0000-0001-7428-7460

Dr. Kushraj Dewan: (1) http://orcid.org/0000-0001-8085-7247

Dr. Gaurav Shrestha: (1) http://orcid.org/0000-0002-1952-7502

Dr. Bhanumati Saikia Patowary: (1) http://orcid.org/0000-0001-5201-3493

Source of Support: Nil, Conflict of Interest: None declared.
} 